
\title{
3 Research Square \\ Drug-Loaded Liposomal Spherical Nucleic Acid as an Effective Cancer Nanovaccine
}

\section{Bo Deng}

Chinese Academy of Medical Sciences \& Peking Union Medical College Institute of Biomedical

Engineering

\section{Bing Ma}

Chinese Academy of Medical Sciences \& Peking Union Medical College

\section{Yingying Ma}

Chinese Academy of Medical Sciences \& Peking Union Medical College Institute of Biomedical

Engineering

Pei Cao

National Center for Nanoscience and Technology

\section{Xigang Leng}

Chinese Academy of Medical Sciences \& Peking Union Medical College

\section{Pengyu Huang}

Chinese Academy of Medical Sciences \& Peking Union Medical College

\section{Tianjiao Ji}

National Center for Nanoscience and Technology

\section{Xueguang Lu ( $\nabla$ xueguang@iccas.ac.cn )}

Institute of Chemistry Chinese Academy of Sciences

\section{Lanxia Liu}

Chinese Academy of Medical Sciences \& Peking Union Medical College Institute of Biomedical Engineering

\section{Research}

Keywords: Nanoparticle, Nanovaccine, In Situ Vaccination, Immunotherapy, Chemotherapy, vaccines, Vaccination

Posted Date: November 17th, 2021

DOI: https://doi.org/10.21203/rs.3.rs-1074689/v1

License: (c) (1) This work is licensed under a Creative Commons Attribution 4.0 International License. Read Full License 


\section{Abstract}

Background: Cancer nanovaccine has become a promising approach for cancer immunotherapy. The major challenge of cancer vaccines is limited efficacy caused by lack of desirable tumor specific antigens (TSA). Chemotherapeutics can trigger immunogenic cell death (ICD) and release TSAs, which initiate tumor-specific immune responses. However, ICD-triggered immune responses are usually not potent enough to eliminate the tumor cells. Herein, we developed liposomal spherical nucleic acids (SNA) that can simultaneously deliver and release doxorubicin (DOX) and $\mathrm{CpG}$ oligonucleotides upon biological stimuli in tumors to augment antitumor immune responses.

Results: SNA nanoparticle increased DOX accumulation at the tumor tissue to induce tumor cells apoptosis and autophagy to activate both ICD-triggered and autophagy-mediated Th1-type immune responses. Meanwhile, CpG, which was co-delivered with DOX, functioned synergistically to potentiate the antitumor immune responses. These nanoparticles effectively inhibited tumor growth and extended animal survival of a mouse lymphoma model.

Conclusions: This work provided a simple strategy of delivering chemotherapeutics and adjuvants to tumors to improve immunotherapeutic efficacy of nanovaccines.

\section{Introduction}

Cancer vaccines harness the immune system to fight against cancer and has become one of the most promising therapies in clinic[1, 2]. An ideal cancer vaccine can boost strong antitumor immune response and avoid severe side effects[3, 4]. Many cancer vaccines that are composed of tumor-associated antigens (TAAs) and adjuvants show promising efficacy on animal models and are currently under clinical investigation $[5,6]$. However, these cancer vaccines could induce strong inflammatory responses due to the non-specificity of antigen. Even though TAAs are highly expressed in tumors, they are still expressed in other healthy organs[7]. Therefore, TAA-specific T cells could attack normal tissue cells and cause severe damage to health organs. Tumor specific antigen (TSA) is preferred to construct cancer vaccines[8]. However, it's highly challenging to construct general TSA because it varies significantly among patients[9].

Selected chemotherapeutics such as doxorubicin (DOX) could trigger immunogenic cell death (ICD)of cancer cells, thus releasing tumor-specific antigens[10,11]. These antigens were recognized by antigenpresenting cells to trigger downstream anticancer immune responses[12]. ICD-triggered immune responses are usually not potent enough to eliminate tumor cells and could be amplified by immunostimulatory reagents, such as $\mathrm{CpG}$ oligodeoxynucleotides (CpG ODN)[13], which can increase the filtration of immune cells into tumor microenvironment $(\mathrm{TME})[14,15]$. Therefore, a variety of studies utilized nanoparticles to co-deliver chemotherapeutics and adjuvants for effective cancer immunotherapy $[16,17]$. However, most nanoparticles are mainly composed of carrier materials such inorganic nanoparticles and polymers $[18,19]$, resulting in low encapsulation efficacy, complexity on the 
preparation and safety evaluation of nanovaccines, and ultimately high barrier for translation[20]. Therefore, a simple system that can efficiently deliver chemotherapeutics and adjuvants to tumors is very much needed.

Herein (Fig. 1), we developed a liposomal hybrid nanoparticle (hNPs) by co-assembly of DOX-1, 2Dioleoyl-sn-glycero-3-phosphoethanolamine (DOPE) conjugate and DOPE-CpG conjugate. Disulfide bond and matrix metalloproteinases-9 (MMP-9)-responsive peptide were incorporated into DOPE-DOX and DOPE-CpG, respectively, for triggered release upon entering tumor microenvironments and tumor cells. This simple nanoparticle design increased drug accumulation in tumor, DC activation efficacy, $C D 8^{+}$and $\mathrm{CD} 4^{+} \mathrm{T}$ cell population, and effectively inhibited tumor growth. Additionally, this nanoparticle reduced the systemic toxicity of DOX, therefore could serve as an effective and safe cancer nanovaccine.

\section{Materials And Methods}

\subsection{Preparation and characterization of DOPE-MMP-CpG and DOPE-DOX}

The hybrid nanoparticle was engineered with two molecules of different compositions and length. The short molecule was DOPE-DOX which was created by the conjugation of Doxorubicin (DOX, Meilunbio, China) with 1,2-Dioleoyl-sn-glycero-3-phosphoethanolamine (DOPE, Sigma-Aldrich Co, USA) by disulfide bond. The long molecule DOPE-MMP-CpG was synthesized with adjuvants molecules CpG-ODN (Type C 2395, sequence: 5'- TCGTCGTTTTCGGCGCGCGCCG-3', Sangon Biotech, China), matrix metalloproteinase9 (MMP-9) responsive peptides (sequence: GPQGIAGQR, ChinaPeptides Co., Ltd, Shanghai) and DOPE.

To synthesize DOPE-DOX, DOPE and DOX were first reacted with N-Succinimidyl 3-(2-pyridyldithio) propionate (SPDP, Thermo Fisher Scientific Inc., USA) at a ratio of 1:1.2 for $8 \mathrm{~h}$ at room temperature, respectively. Then, DL-Dithiothreitol (DTT, Aladdin, China) was added to the pyridyldithiol-activated DOPE and reacted for $3 \mathrm{~h}$ to reduce into sulfhydryl-modified DOPE. Finally, the resulted pyridyldithiol-activated DOX and sulfhydryl-modified DOPE were mixed and stirred for $24 \mathrm{~h}$ at room temperature under the catalysis of triethylamine (TEA), thus DOX was conjugated with DOPE by disulfide bond. The unreacted impurities were removed by $1 \mathrm{kDa}$ MWCO dialysis tubing.

The synthesis of DOPE-MMP-CpG involved four steps. First, MMP-9 responsive peptides were pyridyldithiol-activated by SPDP at a molar ratio of 1:1.2 for $8 \mathrm{~h}$ at room temperature. Then, the resulted pyridyldithiol-activated MMP were reacted with an appropriate amount sulfhydryl-modified CpG-ODN for $24 \mathrm{~h}$ at room temperature and conjugated by disulfide bonds under the presence of TEA. Next, the carboxyl groups of MMP-9 responsive peptides were activated by a suitable amount 1,2-bichloroethane (EDC, Sigma-Aldrich Co., USA) and N-hydro-xysuccinimide (NHS) for $4 \mathrm{~h}$ at $40^{\circ} \mathrm{C}$. At last, the activated carboxyl groups of MMP-9 responsive peptides and the amino groups of DOPE reacted at a ratio of 1:1.2 at room temperature for $24 \mathrm{~h}$ and DOPE-MMP-CpG were synthesized through the amide-forming reaction. The unreacted impurities were removed by 7kDa MWCO dialysis tubing. 
The synthesis of DOPE-MMP-CpG was verified by agarose gel electrophoresis and further determined using Fourier Transform Infrared Spectrometer (FTIS). DOPE-DOX were characterized by FTIS.

\subsection{Preparation and characterization of hNPs}

The DOPE-DOX or DOPE-MMP-CpG with amphiphilic properties was self-assembled itself (named as DOPE-DOX NPs and DMC NPs) or the two molecules self-assembled at ratios of 10:1, 20:1, 30:1 (named as hNPs), respectively. The size and zeta potential of nanoparticles were detected by dynamic light scattering (DLS). The surface morphology was observed by transmission electron microscope (TEM, JEOL JEM-100CX-II, Japan).

To evaluate the responsiveness of hNPs to tumor microenvironment, hNPs were digested by MMP-9 enzyme $(2 \mathrm{ug} / \mathrm{mL})$ for $5 \mathrm{~min}$ and observe their size alteration by DLS. To detect the DOX release behavior from hNPs, nanoparticles were dissolved in PBS with DTT and MMP-9 enzyme in a $1 \mathrm{kDa}$ dialysis tubing and measure the concentration of DOX in dialysate at preset timepoint at $37^{\circ} \mathrm{C}$.

\subsection{In vitro experiments}

\subsubsection{Cytotoxicity assessment}

Human umbilical vein endothelial cells (HUVEC) cultured with endothelial cell medium under $5 \% \mathrm{CO}_{2}$ at $37^{\circ} \mathrm{C}$. In order to evaluate the cytotoxicity of hNPs to HUVEC, HUVEC were seeded into 96-well culture at $10^{4}$ cells/well and co-incubated with hNPs and free DOX at a series of rising concentrations for $24 \mathrm{~h}$. Finally, the in vitro cytotoxicity of hNPs was determined by CCK-8 kit (CCK-8, Dojindo Molecular Technologies, Inc., Japan).

\subsubsection{The uptake of DOX}

In order to assess the uptake and location of DOX in tumor cells, tumor cells E.G7-OVA were co-incubated separately with hNPs and free DOX (DOX concentration was $2 \mu \mathrm{M}$ ) for $2 \mathrm{~h}, 4 \mathrm{~h}$, and $6 \mathrm{~h}$, respectively. After cells were washed with PBS three times, stained with DAPI and fixed using fixative solution for $20 \mathrm{~min}$ in turn, the uptake and location of DOX was observed by Confocal Laser Scanning Microscopy (CLSM, Zeiss LSM 800, Germany) and fluorescence intensity was quantified by ImageJ software.

\subsubsection{BMDC activation and maturation}

Tumor cells E.G7-OVA were treated with PBS, free DOX, free DOX\&CpG and hNPs (DOX concentration was $2 \mu \mathrm{M}, \mathrm{CpG}-\mathrm{ODN}$ concentration was $10 \mu \mathrm{g} / \mathrm{ml}$ ) for $24 \mathrm{~h}$, respectively. Then the treated dying cell or debris were collected and used for the following experiment. Bone Marrow-Derived Dendritic Cells (BMDCs) collected from femur of C57BL/ 6 mice and induced with GM-CSF $(20 \mathrm{ng} / \mathrm{mL})$ and IL-4 $(10 \mathrm{ng} / \mathrm{mL})$ under $5 \% \mathrm{CO}_{2}$ at $37^{\circ} \mathrm{C}$. After a week of cultivation, BMDCs were co-cultured with the above various treated E.G7OVA debris for 48h. After stained with cy5.5-labeled CD11c, FITC-labeled CD86, APC-labeled MHC and PElabeled CD40 antibodies (eBioscience, CA, USA) for 30min. The expression of MHC molecules and costimulating molecules on BMDCs were evaluated with a flow cytometer. In the meantime, cytokines (IL-1ß, 
IFN-y and TNF-a) in the culture supernatant were assessed by ELISA kits (Thermo Fisher Scientific Inc., USA).

\subsection{In vivo immunization experiments 2.4.1 Biodistribution experiment}

In order to detect the biodistribution of hNPs after intravenous injection in mice, DOPE was conjugated with fluorescence cy7 instead of DOX to prepare nanoparticles named as cy7-hNPs. Cy7-hNPs nanoparticles and free cy 7 were injected intravenously $(20 \mu \mathrm{g} / \mathrm{mouse}$ ) into the C57BL/6 mice (Beijing WTLH Laboratory Animal Technology Co., Ltd, Beijing, China), respectively. The in-vivo Maestro imaging system (Maestro EX, USA) was used to monitor the fluorescence signal at various time points in mice. At $48 \mathrm{~h}$ after inject, the mice were sacrificed and tumors and organs were collected for fluorescence imaging and quantified the fluorescence intensity by CRI.

\subsubsection{Therapeutic effect}

All animals were treated under guidelines approved by the animal care and use committee at Peking Union Medical College, China. To establish tumor xenograft models, $5 \times 10^{5}$ E.G7-OVA cells were implanted subcutaneously into the right back of 6-week-old female C57BL/6 mice. Tumor-bearing E.G7-OVA mice were randomized into 4 groups until the volume of tumor reached nearly $50 \mathrm{~mm}^{3}$. Then, mice were injected with PBS, free DOX, free DOX\&CpG and hNPs $(n=6, D O X$ concentration was $0.1 \mathrm{mg} / \mathrm{mouse}$, CpGODN concentration was $80 \mathrm{ng} /$ mouse), respectively, and the treatments were performed 3 times at intervals of 6 days.

To assess the therapeutic efficacy, the body weight of mice, tumor volumes and survival period were recorded every day. Mice were deemed as death when the tumors volume was larger than $2000 \mathrm{~mm}^{3}$ volume.

In order to further evaluate the immune effect of hNPs and explore the related mechanism, mice were sacrificed at 72 hours after the third therapy, and their spleens, hearts, lymph nodes and tumors were harvested.

\subsubsection{Histopathological evaluation of tumor and myocardium}

To evaluate the tumor apoptosis and the cardiovascular toxicity of hNPs. The tumor and heart tissue sections of mice were stained with H\&E (hematoxylin and eosin) for histopathological evaluation.

\subsection{4 $\mathrm{T}$ cell immune responses}

Lymphocytes were isolated from lymph nodes and spleens by lymphocyte separation solution and were processed into single-cell suspension. The lymphocytes were co-cultured with fluorescence-labeled antibodies against CD8, CD4 and CD3 to assess the magnitude of immune response. 


\subsubsection{Cytokine secretion}

Tumor cells were incubated with DOPE-DOX NPs for $24 \mathrm{~h}$ and the dying cells or debris were collected beforehand. $2 \times 10^{5}$ splenic lymphocytes from various treated mice were seeded into 96 -well plates and restimulated with dying cells or debris for $48 \mathrm{~h}$ at $37^{\circ} \mathrm{C}$. After centrifugation at $450 \mathrm{~g}$ for $5 \mathrm{~min}$, the supernatants were harvested to assess the cytokine expression levels of IL-1 $\beta$, IL-18, IFN- $\gamma$ and TNF- $a$ with ELISA kit.

\subsubsection{Immune memory}

Splenic lymphocytes from mice treated with various formulations were re-stimulated with dying tumor cell or debris for 72 hours and stained using different fluorescent CD62L, CD44, CD4 and CD8 antibodies (eBioscience, CA, USA). The proliferation of memory T cells were detected with flow cytometry.

\subsection{Statistical analysis}

All data were presented as mean result \pm standard deviation (SD). Statistical significance of differences was analyzed using Student's t-test or ANOVA analysis. P-value less than 0.05 was considered as statistically significant.

\section{Results And Discussion}

\section{1. hNPs preparation and characterization}

DOPE-MMP-CpG was composed of DOPE, MMP-9 responsive peptide and CpG-ODN. Successful synthesis of DOPE-MMP-CpG was proved by Fourier-transform infrared spectroscopy (FT-IR) (Fig. 2A). There was a new absorption peak at $531 \mathrm{~cm}^{-1}$ and $534 \mathrm{~cm}^{-1}$ of MMP-CpG and DOPE-MMP-CpG, respectively, which was attributed to the disulfide bond (S-S) of CpG-MMP formed from sulfhydryl group of CpG-SH and pyridyldithiol-activated MMP. Additionally, DOPE contains a large amount of methylene, a newly appeared peak for methylene $\left(\mathrm{CH}_{2}\right)$ at $2915 \mathrm{~cm}^{-1}$ and $2853 \mathrm{~cm}^{-1}$ in DOPE-MMP-CpG proved that DOPE covalently connected to MMP-CpG successfully. The successful synthesis of DOPE-MMP-CpG was further confirmed by $2 \%$ agarose gel electrophoresis (Fig. 2B).

DOPE-DOX was synthesized by coupling DOPE with DOX through a SPDP linker. After the unreacted impurities were removed by dialysis, the conjugate of DOPE-S-S-DOX was characterized with FT-IR. As shown in Fig. 2C, compared with free DOX, the absorption peak of DOPE-S-S-DOX at $2852 \mathrm{~cm}^{-1}$ and 2923 $\mathrm{cm}^{-1}$ was designated to the $\mathrm{CH}_{2}$ stretching vibration. In the spectrum of DOPE-S-S-DOX, a new absorption peak emerged at $462 \mathrm{~cm}^{-1}$, which is the characteristic absorption peak of disulfide bonds. These results indicated that DOPE-DOX had been successfully conjugated by disulfide bond.

Different ratios of DOPE-MMP-CpG and DOPE-DOX were allowed to self-assemble in water to form nanoparticles (hNPs). The hydrodynamic diameter and size distribution of nanoparticles were measured 
by DLS. As shown in Fig. S1, DOPE-DOX or DOPE-MMP-CpG could self-assemble into nanoparticles by themselves. They could also form stable hybrid nanoparticles (hNPs) at different ratios. According to the dose of DOX and CpG ODN in the following in vivo experiments, the hNPs at the ratio of 30:1 (DOPE-DOX: DOPE-MMP-CpG) was prepared for following experiments. The diameter of these hNPs was $~ 160.6 \pm 1.3$ $\mathrm{nm}(\mathrm{PDI} 0.126 \pm 0.05)$ and the Zeta potential was $-25.3 \pm 0.9 \mathrm{mV}$. TEM showed that hNPs exhibit spherical morphology with uniform size dispersion (Fig. 2D). As shown in Fig. S2 and Fig. S3, hNPs could remain stable for three weeks in PBS and one week in RPMI medium containing 10\% FBS, respectively. These results demonstrated that DOPE-DOX and DOPE-MMP-CpG could form stable hybrid nanoparticles with an inside small spherical DOX and an outside large spherical nucleic acid (CpG ODN).

To evaluate the MMP-9-mediated responsiveness of hNPs, the size alteration of hNPs was observed before and after incubation with MMP-9 enzyme. The diameter of NPs became clearly smaller after incubation with MMP-9 and was highly similar to that of DOPE-DOX NPs (Fig. 2E), indicating that hNPs released CpG ODN upon peptide cleavage. Moreover, the remained DOPE-DOX maintained stable as nanoparticles. These results suggested that hNPs could be cleaved in the tumor microenvironment with a high level of MMP-9 enzyme and release CpG ODN to stimulate antigen presenting cells.

The release of DOX from hNPs was evaluated in PBS containing $0.01 \mathrm{M}$ DTT to mimic the high intracellular reducing environment of tumor cells. The results showed that $\sim 34.1 \%$ of DOX burst released in the first 3 days, and then constantly and slowly released reaching up to $59.9 \pm 2.5 \%$ over 31 days (Fig. 2F). This sustained DOX-release profile suggested that DOPE-DOX NPs could kill tumor cells for a long period of time.

\subsection{In vitro experiments 3.2.1 Cytotoxicity assessment}

We first assessed whether hNPs could induce less cytotoxicity against normal endothelium cells than free DOX. The HUVEC were treated with hNPs at different concentrations for 24 hours. Cell viability was evaluated by CCK-8 assay. No obvious cytotoxicity was observed for hNPs-treated cells even at high concentrations (up to $2 \mathrm{mM}$, Fig. 3A). On the other hand, free DOX decreased cell viability to $\sim 40 \%$ at the same concentration, indicating that hNPs possessed better safety profile than free DOX.

\subsubsection{Uptake of DOX}

When hNPs reached at the tumor site where MMP-9 enzyme highly expressed, these nanoparticles would release the outside CpG ODN to stimulate immune responses and the remained DOPE-DOX NPs would kill tumor cells. Several studies demonstrated that free DOX and nano-DOX differed in their subcellular distribution and the way they caused tumor cell death[21, 22]. Free DOX intercalated into DNA in nuclei resulting in cell oxidative damage and induced ICD-triggered immune responses. Nano-DOX primarily stayed in the cytoplasm and induced mutual reinforced loop between autophagy and release HMGB1, which can elicit powerful immune responses. We studied the cellular uptake of DOPE-DOX NPs in a tumor 
cell line E.G7-OVA. Free DOX or DOPE-DOX NPs were incubated with E.G7-OVA cells for different periods of time before imaging by CLSM. The amounts of cellular uptake between free DOX and DOPE-DOX NPS were similar at $6 \mathrm{~h}$ post incubation (Fig. 3B). However, the intracellular distribution of DOX is highly different. Most of DOPE-DOX NPs appeared in cytoplasm. Most of free DOX located in nuclei (Fig. 3C). The results of intracellular DOX distribution combined with the profile of DOX-release indicated that DOPE-DOX NPs could induce both ICD and autophagy-triggered immune responses.

\subsubsection{BMDC activation and maturation}

The maturation and activation of dendric cells (DCs) is the key to initiate strong immune responses. We first evaluated whether tumor cells treated with hNPs could induce DC maturation and activation. To mimic the process in tumor microenvironment, BMDCs from mice were incubated for 48h with E.G7-OVA cells, which were pre-treated with hNPs (treated with MMP-9 enzyme beforehand), free DOX\&CpG, or free DOX for $24 \mathrm{~h}$, respectively. DOX concentration in each group was $2 \mu \mathrm{M}, \mathrm{CpG}-\mathrm{ODN}$ concentration was $10 \mu \mathrm{g} / \mathrm{ml}$. E.G7-OVA treated with PBS was utilized as the negative control. Then BMDCs were labeled with antibodies against CD11C, MHC-II and CD40 and evaluated by flow cytometry. Cytokines (IL-1 $\beta$, IFN- $\gamma$ and TNF-a) levels in the culture supernatant were assessed by ELISA kits. The results showed that the expression of co-stimulatory molecules CD40 and MHC-II in the hNPs group were dramatically enhanced compared with free DOX\&CpG and free DOX (Fig. 3D and 3E), indicating that hNPs could promote BMDCs maturation. The cytokine secretion of IFN- $\gamma$ and IL-1 $\beta$ by DCs in hNPs group was significantly enhanced compared with other groups (Fig. $3 \mathrm{G}$ and $3 \mathrm{H}$ ). The secretion of TNF-a, which play a crucial role in the proliferation of T cells, were elevated over $200 \%$ by hNP treatment in comparison to PBS group (Fig. 3F), indicating hNPs remarkably elicit and amply the immune responses. These results suggested that hNPs could effectively induce ICD of tumor cells and activated the NOD-like receptor family, pyrin domain containing - 3 protein (NLRP3) - dependent (inflammasome) pathway[23] and TLR-CpG ODN pathway[24] in DC which both facilitate tumor-specific IFN- $y$ to produce $\mathrm{T}$ cell immune responses. All the above results clearly demonstrated that hNPs could provide an effective immune stimulus and facilitate DCs maturation and activation to invoke tumor-specific immune responses.

\subsection{In vivo immunization experiments 3.3.1 Biodistribution of hNPs}

Systemic administration of free DOX not only cause off-target toxic effects, but also reduce the local concentration of drugs at the tumor site accompanied by weak induction of ICD. Nanoparticles are expected to offer superior accumulation in local tumor tissues and less adverse side effects than conventional chemotherapeutic drugs[25-27]. To determine whether these carrier-free hNPs could promote drug accumulation at tumor site after intravenous injection, fluorescence cy 7 instead of DOX was used to construct hybrid nanoparticles (cy7-hNPs). Cy7-hNPs were administered into tumor bearing mice by intravenous injection. The biodistribution of cy7-hNPs in tumor-bearing mice was imaged using the Maestro imaging system. The results showed that the cy7-hNPs continuously increased at tumor site 
within $24 \mathrm{~h}$ after injection and the fluorescent intensity still kept comparatively high at $48 \mathrm{~h}$ compared to free drugs (Fig. 4A).

At $48 \mathrm{~h}$ after intravenous injection, the mice were sacrificed, and tumors and organs were collected for ex vivo fluorescence imaging to quantify the biodistribution of cy7-hNPs. As shown in Fig. 4B and 4C, free cy7 group showed strong fluorescence signal in the kidneys and lungs, but weak signal in tumors. On the contrary, cy7-hNPs group showed much more fluorescence signal in tumors and fewer signal in normal tissue. These results proved that hNPs altered the biodistribution of DOX and increased drugs accumulation in the tumor.

\subsubsection{The therapeutic effect of hNPs}

To further estimate the in vivo tumor inhibition effect of hNPs, an E.G7-OVA tumor model was established and treated with various formulations. The results of survival curves and tumor volume changes were shown in Fig. 4D and 4E. After three times of administration, hNPs group showed more potent tumor inhibition effect compared with other groups. Furthermore, the average survival of mice treated with PBS, free DOX and free DOX\&CpG were 21.4d, 24.2d, and 24.4d, respectively. hNPs significantly exceed survival period. $80 \%$ of mice treated with hNPs survived over 30 days and $40 \%$ survived over 45 days. Moreover, hNPs showed less toxicity compared to free DOX and DOX\&CpG groups. Mice treated with free DOX or free DOX\&CpG showed significant weight loss and displayed a depressed mental status (Fig. S5). But the body weight of mice treated with hNPs remained constant and exhibited positive mental states. The results of H\&E staining further confirmed that hNPs induced clear apoptosis in tumor tissue with reduced cardiovascular toxicity compared to free DOX and free DOX\&CpG (Fig. 4F). These results demonstrated that hNPs exhibited prominent anti-tumor effect with excellent safety.

\subsubsection{Analysis of T cells immune activation}

To further evaluate the activation of immune responses, we assessed the proliferation of lymphocytes in lymph nodes and spleen after treatments with hNPs, free DOX, free DOX\&CpG, and PBS, respectively. Compared to PBS, the percentage of $\mathrm{CD}^{+} \mathrm{CD}^{+} \mathrm{T}$ cells in lymph nodes treated with hNPs enhanced $\sim 3$ times, from $6.3-18.2 \%$ (Fig. 5A and 5B). The percentage of $\mathrm{CD}^{+}{ }^{+} \mathrm{CD} 3^{+}$significantly enhanced $\sim 9$ times, from 1.5-13.1\% (Fig. 5C and 5D). The lymphocytes in spleen also showed significant enhanced

proliferation (Fig. $5 \mathrm{E}-5 \mathrm{H}$ ). The percentages of $\mathrm{CD} 4^{+} \mathrm{CD}^{+} \mathrm{T}$ cells and $\mathrm{CD} 8^{+} \mathrm{CD} 3^{+} \mathrm{T}$ cells reached $31.3 \%$ and $16.9 \%$ in hNPs group, respectively, displaying $\sim$-fold compared to PBS group. These results indicated that hNPs promoted proliferation of both $\mathrm{CD} 4^{+} \mathrm{CD} 3^{+} \mathrm{T}$ cells and $\mathrm{CD} 8^{+} \mathrm{CD} 3^{+} \mathrm{T}$ cells.

\subsubsection{Mechanism analysis}

Previous studies showed that free DOX could serve as an apoptosis inducer and initiates ICD-inducing immune responses through inflammasome pathway. Nano-DOX induced autophagy releasing HMGB1 which could promote Th1-type immune responses[28]. In addition, C-type CpG ODN, as TLR9 agonists, could amplify innate and adaptive T-cell immune responses and release cytokines including TNF-a, IFN- $\gamma$, 
et al, which could further enhance the anti-tumor immunity. To further verify how hNPs could enhance DOX-triggered immune responses and explore the underlying mechanism, the lymphocytes in spleens after various treatments were collected and restimulated with E.G7-OVA fragments for 72 hours. The expression level of inflammasome pathway-related cytokines (IL-1 $\beta$ and IL-18) and Th1-type cytokines (TNF- $\alpha$ and IFN- $\gamma$ ) in cell culture supernatant were detected. As shown in Fig. 6A-5D, hNPs remarkably improved the level of IL-1 $\beta$ and IL-18 and increased the secretion of TNF- $\alpha$ and IFN- $\gamma$ compared to free DOX and free DOX\&CpG treated groups. The level of TNF- $\alpha$ and IFN- $\gamma$ was enhanced by $\sim 5$ and $\sim 14$ times higher than PBS group, respectively. Collectively, these results suggested that hNPs could significantly enhance tumor cells apoptosis and autophagy and activate both ICD-induced immune responses and Th1-type immune responses.

\subsubsection{Memory immunity}

Encouraged by the potent systemic immune response of hNPs in lymph nodes and spleen, which could inhibit the tumor metastasis, we evaluated the induction of central memory T cell $\left(T c m, C D 62 L^{\text {hi }} C D 44^{+} T\right.$ cell)[29]. Tcm could persist for a long duration and have a rapid recall ability to recognize old-antigens and prime antigen-specific immune responses to prevent tumor recurrence[30]. The lymphocytes from the spleen of immunized mice were re-stimulated with E.G7-OVA fragments. The cells were stained with $\mathrm{CD} 62 \mathrm{~L}$ and CD44 antibodies and detected with FACS. The results demonstrated that hNPs induced remarkable expansion of both $\mathrm{CD} 4^{+} \mathrm{Tcm}$ and $\mathrm{CD} 8^{+} \mathrm{Tcm}$. The percentages of $\mathrm{CD} 4^{+} \mathrm{Tcm}$ in hNPs group was enhanced to $24.7 \%$ compared to PBS group (6.3\%), free DOX (9.0\%), and free DOX\&CpG $(12.5 \%)$ (Fig. 6E and 6F). hNPs also remarkedly elevated the proliferation of $\mathrm{CD} 8^{+} \mathrm{Tcm}$ compared to other groups (hNPs $19.2 \%$ vs PBS $5.1 \%$ vs free DOX 6.4\% vs free DOX\&CpG 8.5\%) (Fig. $6 \mathrm{G}$ and $6 \mathrm{H}$ ). These results indicated that hNPs could induce anti-tumor memory immunity, therefore holding a potential of inhibiting tumor recurrence for a long period.

\section{Conclusion}

In summary, we successfully designed MMP-9/redox responsive nano-delivery system with DOX and CpG-ODN. The hybrid nano-delivery system could sequentially release DOX and CpG-ODN from the nanoparticle at tumor site. It could not only directly induce tumor cells apoptosis and autophagy, but also activate both ICD-induced immune responses and autophagy mediated Th1-type immune responses. Meanwhile, hNPs enhanced the DOX-triggered personalized immune responses to further eliminate tumor cells. Our system increases DC activation efficacy, CD $8^{+}$and $C D 4^{+} T$ cell population in TME, effectively inhibited tumor growth, and extended animal survival. Additionally, this nanoparticle reduced the systemic toxicity of DOX, therefore could serve as an effective and safe cancer nanovaccine. Overall, this work provided a simple design strategy of cancer nanovaccines for effective delivery of both chemotherapeutics and adjuvants.

\section{Declarations}


Availability of data and materials

All date generated or analyzed during this research are included in this published article.

\section{Acknowledgements}

This work was supported by the Natural Science Foundation of China (31870920), the CAMS Innovation Fund for Health and Longevity pilot project (Youth Award Program, 2019-RCHL-015), the CAMS basic research project (2020-RC310-007) and Fundamental Research Funds for the Central Universities (2019PT320028).

\section{Funding}

Natural Science Foundation of China (31870920); CAMS Innovation Fund for Health and Longevity pilot project (2019-RCHL-015); CAMS basic research project (2020-RC310-007); Fundamental Research Funds for the Central Universities (2019PT320028)

\section{Author information}

Affiliations

Tianjin Key Laboratory of Biomedical Materials, Key Laboratory of Biomaterials and Nanotechnology for Cancer Immunotherapy, Institute of Biomedical Engineering, Chinese Academy of Medical Sciences \& Peking Union Medical College, Tianjin 300192, China

Bo Deng, Bing Ma, Yingying Ma, Xigang Leng, Pengyu Huang \& Lanxia Liu

CAS Key Laboratory for Biomedical Effects of Nanomaterials \& Nanosafety, CAS Center for Excellence in Nanoscience, National Center for Nanoscience and Technology, Beijing 100190, China

Pei Cao \& Tianjiao Ji

Key Laboratory of Colloid, Interface and Chemical Thermodynamics, Institute of Chemistry, Chinese Academy of Science, Beijing100190, PR China.

Xueguang Lu

Corresponding author

Correspondence to Xueguang Lu or Lanxia Liu

Contributions

BD designed and performed the most of experiments, analysed the data and did initial draft write-up. BM assisted with In vivo immunization experiments. YYM and PC assisted with BMDC activation and maturation experiments and analyzed part of the data. XGL and PYH contributed to the study concept 
and data analysis. TJJ contributed to discussion, reviewed and edited the manuscript. XGL and LXL conceived and supervised the study, modified and finalized the manuscript.

\section{Ethics declarations}

Ethics approval and consent to participate

All animals were treated under guidelines approved by the animal care and use committee at Peking Union Medical College, China.

Consent for publication

All authors agreed to publish this manuscript.

Competing interests

No conflict of interest exists in this manuscript.

\section{References}

1. Sahin U; Oehm P; Derhovanessian E; Jabulowsky R A; Vormehr M; Gold M, et al. An RNA vaccine drives immunity in checkpoint-inhibitor-treated melanoma. Nature. 2020;585 (7823):107112.https://doi.org/10.1038/s41586-020-2537-9.

2. Bol K F; Schreibelt G; Rabold K; Wculek S K; Schwarze J K; Dzionek A, et al. The clinical application of cancer immunotherapy based on naturally circulating dendritic cells. Journal for immunotherapy of cancer. 2019;7 (1):109.https://doi.org/10.1186/s40425-019-0580-6.

3. Aikins M E; Xu C; Moon J J. Engineered Nanoparticles for Cancer Vaccination and Immunotherapy. Accounts of chemical research. 2020;53 (10):2094-

2105.https://doi.org/10.1021/acs.accounts.0c00456.

4. Janikashvili N; LaCasse C J; Larmonier C; Trad M; Herrell A; Bustamante S, et al. Allogeneic effector/memory Th-1 cells impair FoxP3+ regulatory $T$ lymphocytes and synergize with chaperonerich cell lysate vaccine to treat leukemia. Blood. 2011;117 (5):155564.https://doi.org/10.1182/blood-2010-06-288621.

5. Li W H; Li Y M. Chemical Strategies to Boost Cancer Vaccines. Chemical reviews. 2020;120 (20):11420-11478.https://doi.org/10.1021/acs.chemrev.9b00833.

6. Das K; Belnoue E; Rossi M; Hofer T; Danklmaier S; Nolden T, et al. A modular self-adjuvanting cancer vaccine combined with an oncolytic vaccine induces potent antitumor immunity. Nature communications. 2021;12 (1):5195.https://doi.org/10.1038/s41467-021-25506-6.

7. Melero I; Grimaldi A M; Perez-Gracia J L; Ascierto P A. Clinical development of immunostimulatory monoclonal antibodies and opportunities for combination. Clinical cancer research: an official 
journal of the American Association for Cancer Research. 2013;19 (5):9971008.https://doi.org/10.1158/1078-0432.Ccr-12-2214.

8. Peng M; Mo Y; Wang Y; Wu P; Zhang Y; Xiong F, et al. Neoantigen vaccine: an emerging tumor immunotherapy. Molecular cancer. 2019;18 (1):128.https://doi.org/10.1186/s12943-019-1055-6.

9. Sahin U; Türeci Ö. Personalized vaccines for cancer immunotherapy. Science (New York, N.Y.). 2018;359 (6382):1355-1360.https://doi.org/10.1126/science.aar7112.

10. Xie L; Wang G; Sang W; Li J; Zhang Z; Li W, et al. Phenolic immunogenic cell death nanoinducer for sensitizing tumor to PD-1 checkpoint blockade immunotherapy. Biomaterials. 2021;269:120638.https://doi.org/10.1016/j.biomaterials.2020.120638.

11. Yang W; Zhu G; Wang S; Yu G; Yang Z; Lin L, et al. In Situ Dendritic Cell Vaccine for Effective Cancer Immunotherapy. ACS nano. 2019;13 (3):3083-3094.https://doi.org/10.1021/acsnano.8b08346.

12. Kroemer G; Galluzzi L; Kepp O; Zitvogel L. Immunogenic cell death in cancer therapy. Annual review of immunology. 2013;31:51-72.https://doi.org/10.1146/annurev-immunol-032712-100008.

13. Xia C; Yin S; Xu S; Ran G; Deng M; Mei L, et al. Low Molecular Weight Heparin-Coated and DendrimerBased Core-Shell Nanoplatform with Enhanced Immune Activation and Multiple Anti-Metastatic Effects for Melanoma Treatment. Theranostics. 2019;9 (2):337354.https://doi.org/10.7150/thno.29026.

14. Deng C; Zhang Q; Jia M; Zhao J; Sun X; Gong T, et al. Tumors and Their Microenvironment DualTargeting Chemotherapy with Local Immune Adjuvant Therapy for Effective Antitumor Immunity against Breast Cancer. Advanced science (Weinheim, Baden-Wurttemberg, Germany). 2019;6 (6):1801868.https://doi.org/10.1002/advs.201801868.

15. Kranz L M; Diken M; Haas H; Kreiter S; Loquai C; Reuter K C, et al. Systemic RNA delivery to dendritic cells exploits antiviral defence for cancer immunotherapy. Nature. 2016;534 (7607):396401.https://doi.org/10.1038/nature18300.

16. Riley R S; June C H; Langer R; Mitchell M J. Delivery technologies for cancer immunotherapy. Nature reviews. Drug discovery. 2019;18 (3):175-196.https://doi.org/10.1038/s41573-018-0006-z.

17. Bolhassani A; Safaiyan S; Rafati S. Improvement of different vaccine delivery systems for cancer therapy. Molecular cancer. 2011;10:3.https://doi.org/10.1186/1476-4598-10-3.

18. Minelli C; Lowe S B; Stevens M M. Engineering nanocomposite materials for cancer therapy. Small (Weinheim an der Bergstrasse, Germany). 2010;6 (21):233657.https://doi.org/10.1002/smll.201000523.

19. Paris J L; Baeza A; Vallet-Regí M. Overcoming the stability, toxicity, and biodegradation challenges of tumor stimuli-responsive inorganic nanoparticles for delivery of cancer therapeutics. Expert opinion on drug delivery. 2019;16 (10):1095-1112.https://doi.org/10.1080/17425247.2019.1662786.

20. Escalante J; McQuade R M; Stojanovska V; Nurgali K. Impact of chemotherapy on gastrointestinal functions and the enteric nervous system. Maturitas. 2017;105:2329.https://doi.org/10.1016/j.maturitas.2017.04.021. 
21. Li T F; Li K; Wang C; Liu X; Wen Y; Xu Y H, et al. Harnessing the cross-talk between tumor cells and tumor-associated macrophages with a nano-drug for modulation of glioblastoma immune microenvironment. Journal of controlled release: official journal of the Controlled Release Society. 2017;268:128-146.https://doi.org/10.1016/j.jconrel.2017.10.024.

22. Chen Z; Wang C; Li T F; Li K; Yue Y; Liu X, et al. Doxorubicin conjugated with nanodiamonds and in free form commit glioblastoma cells to heterodromous fates. Nanomedicine (London, England). 2019;14 (3):335-351.https://doi.org/10.2217/nnm-2018-0330.

23. Ghiringhelli F; Apetoh L; Tesniere A; Aymeric L; Ma Y; Ortiz C, et al. Activation of the NLRP3 inflammasome in dendritic cells induces IL-1 beta-dependent adaptive immunity against tumors. Nature medicine. 2009;15 (10):1170-8.https://doi.org/10.1038/nm.2028.

24. Krieg A M. Therapeutic potential of Toll-like receptor 9 activation. Nature reviews. Drug discovery. 2006;5 (6):471-84.https://doi.org/10.1038/nrd2059.

25. Shi Y; van der Meel R; Chen X; Lammers T. The EPR effect and beyond: Strategies to improve tumor targeting and cancer nanomedicine treatment efficacy. Theranostics. 2020;10 (17):79217924.https://doi.org/10.7150/thno.49577.

26. Maeda H; Nakamura H; Fang J. The EPR effect for macromolecular drug delivery to solid tumors: Improvement of tumor uptake, lowering of systemic toxicity, and distinct tumor imaging in vivo. Advanced drug delivery reviews. 2013;65 (1):71-9.https://doi.org/10.1016/j.addr.2012.10.002.

27. Ji T; Kohane D S. Nanoscale systems for local drug delivery. Nano today. 2019;28.https://doi.org/10.1016/j.nantod.2019.100765.

28. Kansal S; Tandon R; Verma A; Misra P; Choudhary A K; Verma R, et al. Coating doxorubicin-loaded nanocapsules with alginate enhances therapeutic efficacy against Leishmania in hamsters by inducing Th1-type immune responses. British journal of pharmacology. 2014;171 (17):403850.https://doi.org/10.1111/bph.12754.

29. Mueller S N; Gebhardt T; Carbone F R; Heath W R. Memory T cell subsets, migration patterns, and tissue residence. Annual review of immunology. 2013;31:137-61.https://doi.org/10.1146/annurevimmunol-032712-095954.

30. Mueller S N; Mackay L K. Tissue-resident memory T cells: local specialists in immune defence. Nature reviews. Immunology. 2016;16 (2):79-89.https://doi.org/10.1038/nri.2015.3.

\section{Figures}

\section{Figure 1}

Schematic illustration of preparation and mechanistic action of hNPs. 

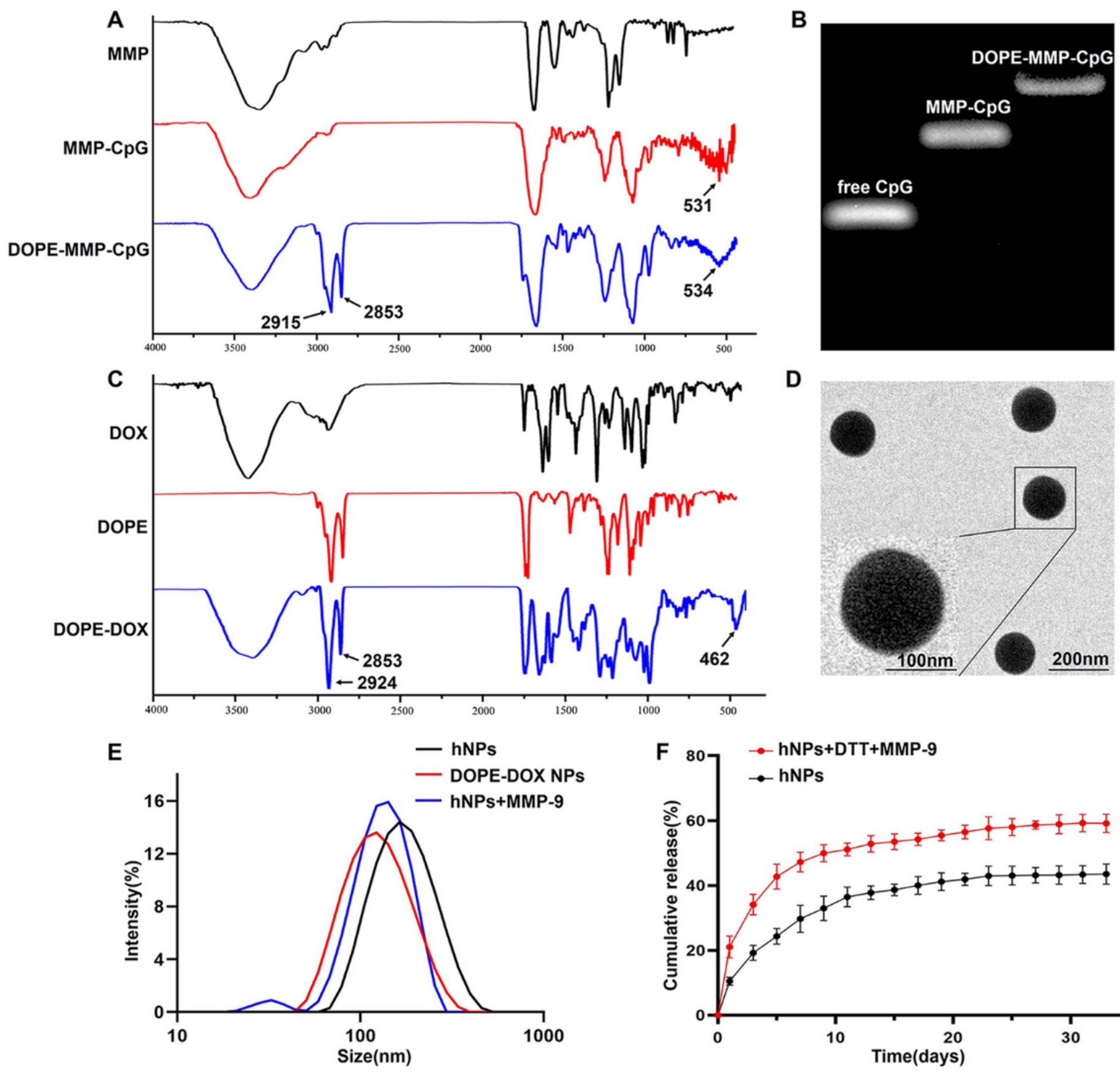

D
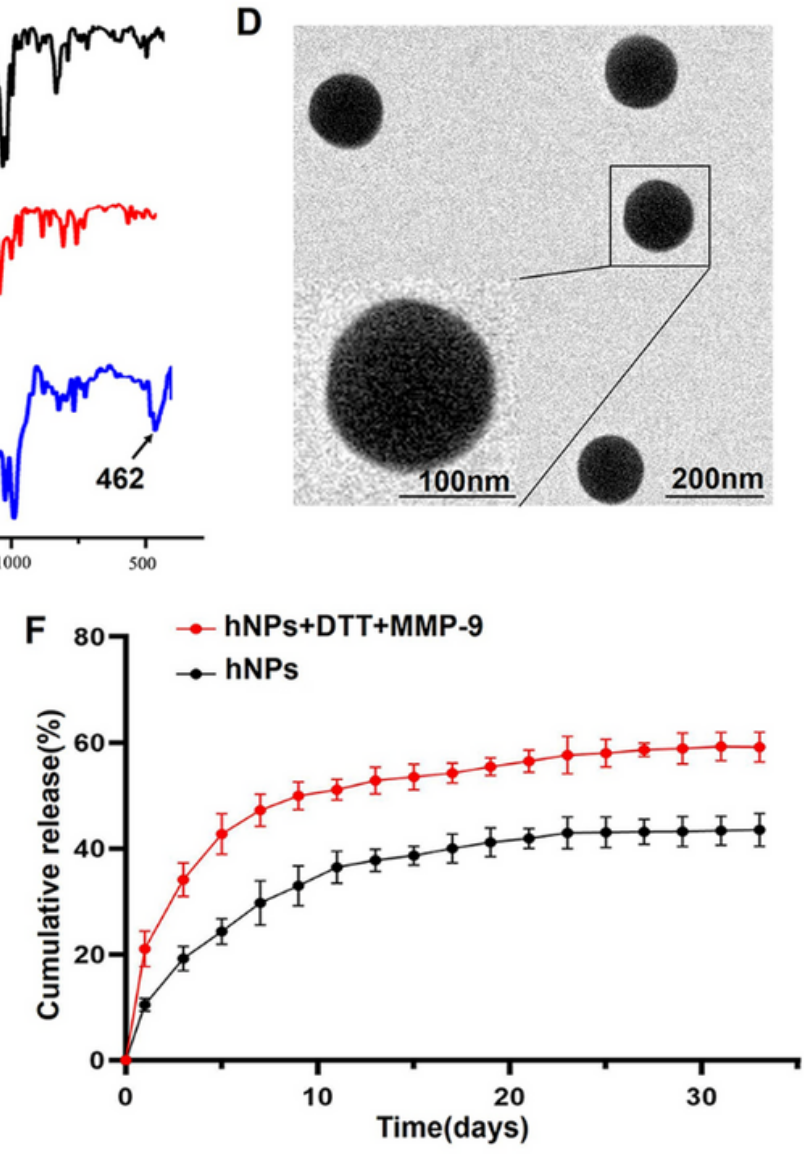

Figure 2

Characterization of nanomaterials and nanoparticles. (A) FTIR measurements of MMP responsive peptide (MMP), MMP-CpG, and DOPE-MMP-CpG. (B) Synthesis of DOPE-MMP-CpG was confirmed by agarose gel electrophoresis. (C) The results of FTIR of DOX, DOPE and DOPE-DOX. (D) Representative TEM images of hNPs. (E) DLS measurements of hNPs before and after incubation with MMP-9 enzyme. DOPE-DOX NPs were used as a control. (F) DOX release profile of hNPs in PBS without/with MMP-9 enzyme and DTT. 
A

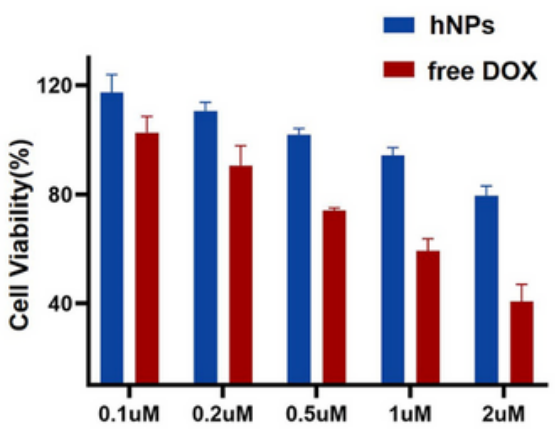

C

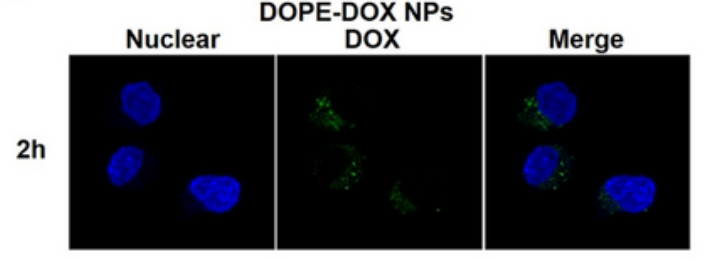

4h

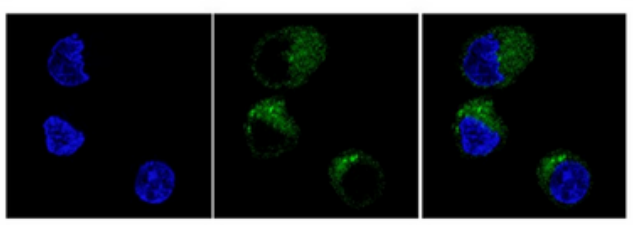

$6 \mathrm{~h}$

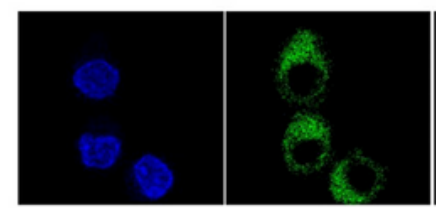

D

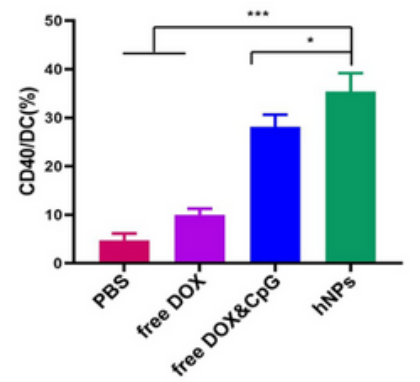

G
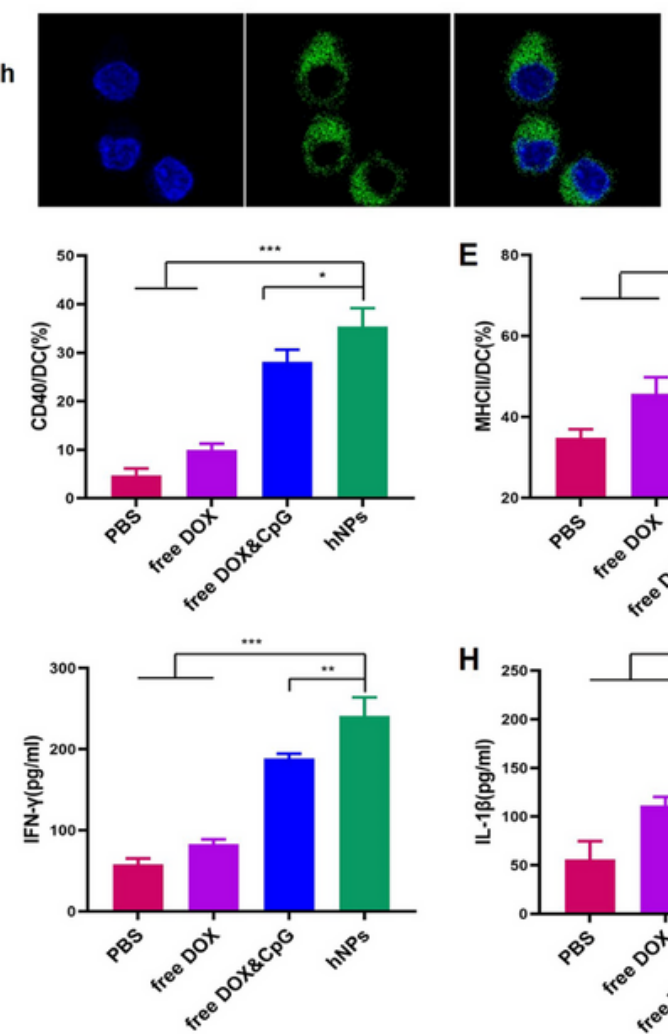
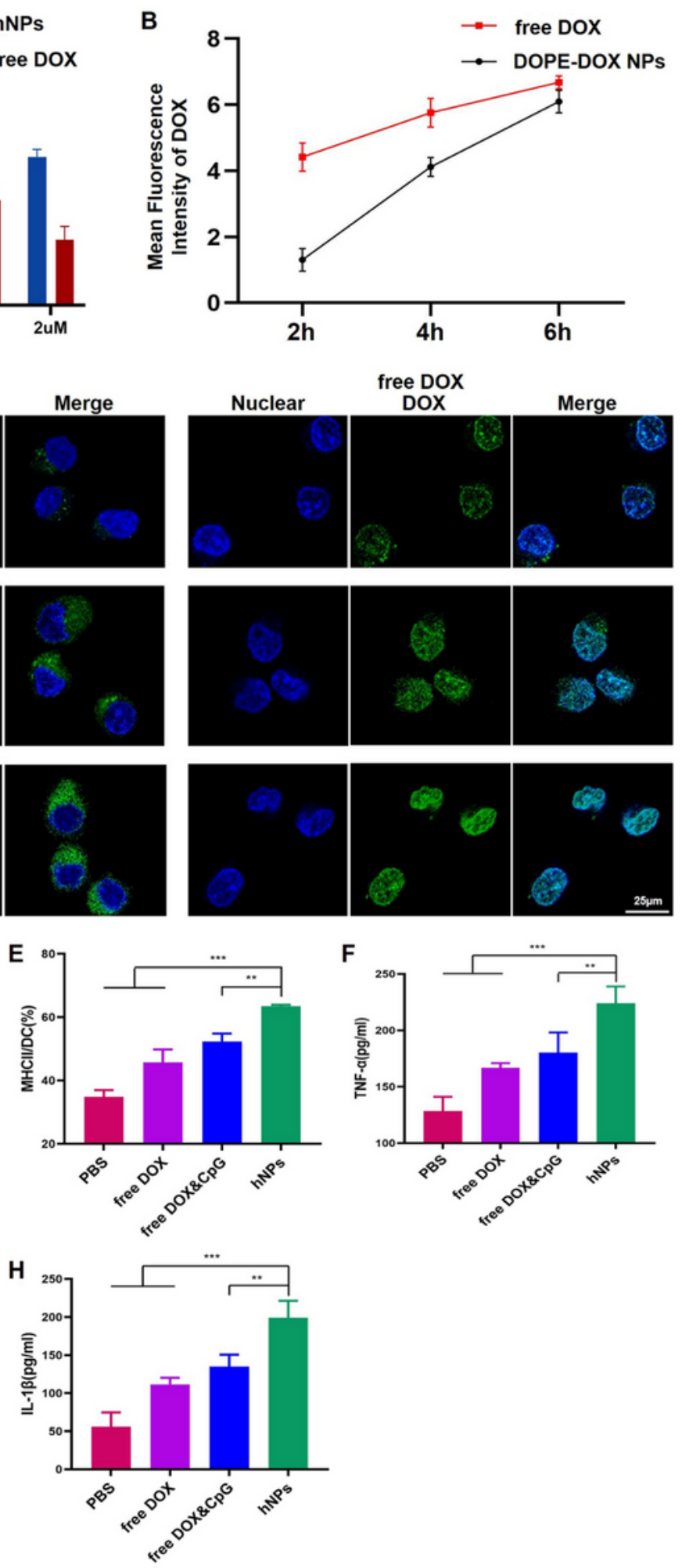

\section{Figure 3}

(A) Cell viability of HUVEC after incubation with various concentrations of hNPs or free DOX\&CpG. (B) Fluorescence intensity of DOX uptake by E. G7 were quantified by Image J. (C) Representative CLSM images of E. G7 after co-incubated with hNPs and free DOX for $2 \mathrm{~h}, 4 \mathrm{~h}$ and $6 \mathrm{~h}$, respectively. The molecule expression of (D) CD40 and (E) MHC-II was analyzed by flow cytometry. The cytokine expression of (F) 
TNF-a, (G) IFN- $y$ and $(H)$ IL-1 $\beta$ were analyzed with ELISA assays. Results represent mean \pm SD $(n=6$; *P< $0.05, * * P<0.01, * \star * P<0.001)$.

\section{Figure 4}

The biodistribution and therapeutic effect of hNPs in E.G7-OVA tumor-bearing mice. (A) Typical fluorescence images of mice at selected time-points after treatment with cy7-hNPs and free cy7 by intravenous injection. (B)The fluorescence images of organs and tumors collected from mice at $48 \mathrm{~h}$ after injection and (C) mean fluorescence intensity were shown. (D) Survival curves of mice treated with various formulations. (E) Changes of tumor volume after mice treated with PBS, free DOX, free DOX\&CpG and hNPs, respectively. (F) H\&E staining images of tumor and heart tissues from mice treated with various formulations. Results represent mean $\pm S D(n=6 ; * P<0.05, * \star \star P<0.001)$. 
A

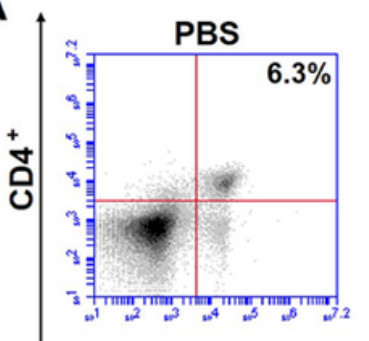

C

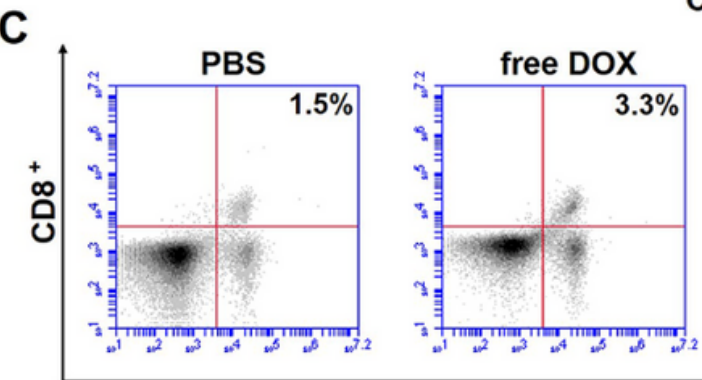

E

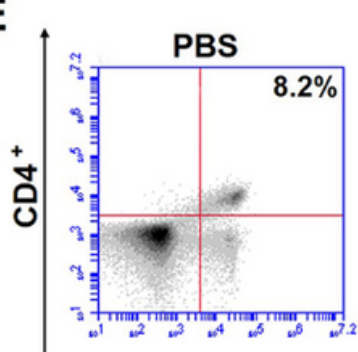

G

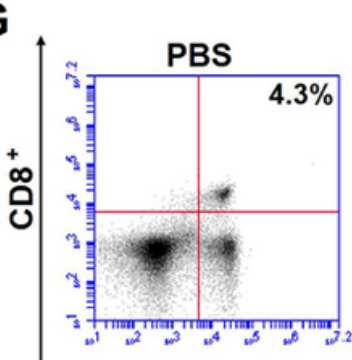

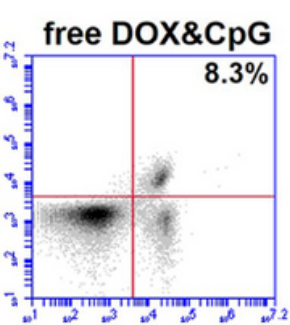

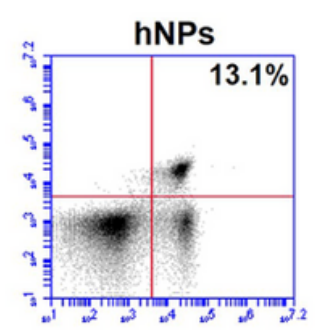

$\mathrm{CD}^{+}{ }^{+}$
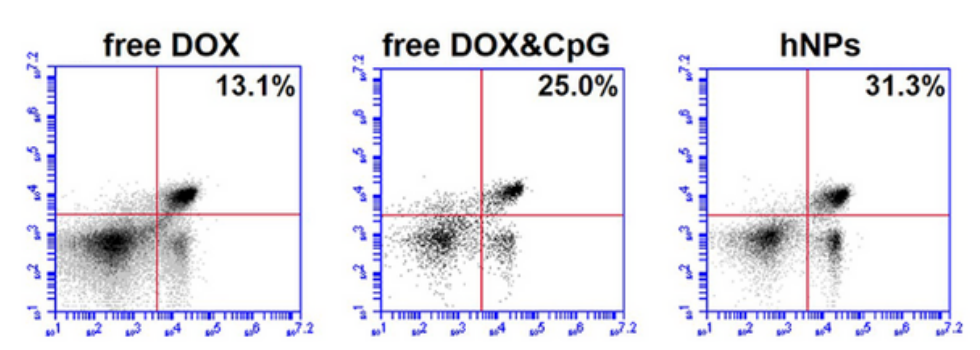

CD ${ }^{+}$

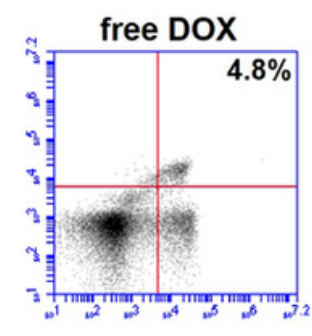
hNPs
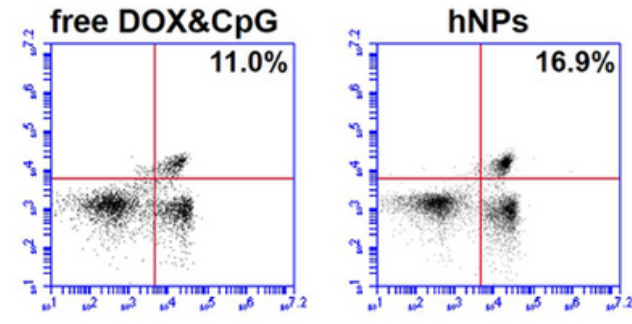
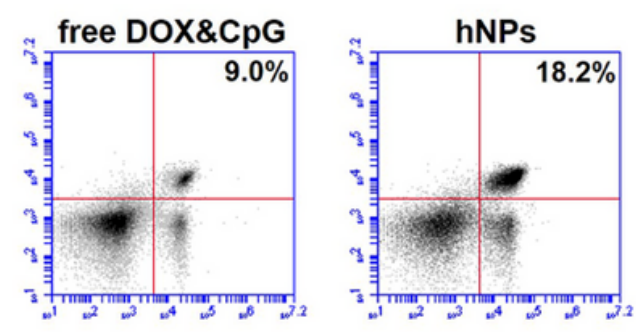

CD3 ${ }^{+}$

B

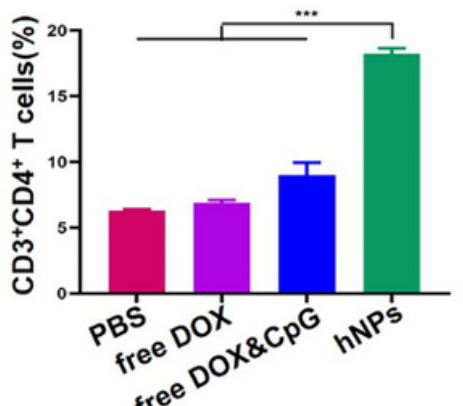

D

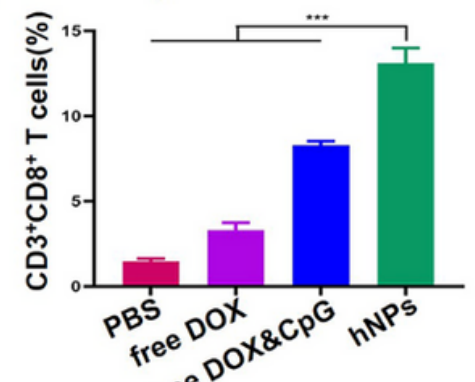

$F$

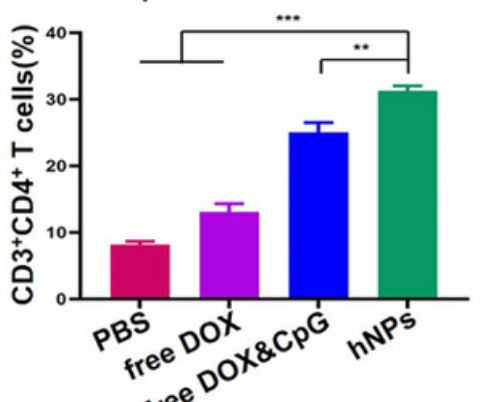

H

$\mathrm{CD}^{+}$

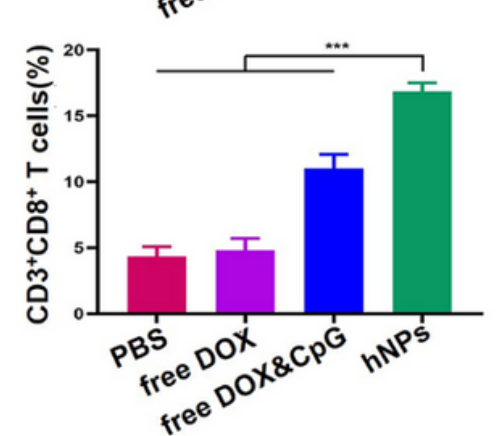

Figure 5

hNPs effectively enhanced proliferation of CD3+CD4+ and CD3+CD8+ T cells. (A) Representative FACS plots and (B) Percentages of CD3+CD4+ and CD3+CD8+ (C and D) T cells in lymph nodes. (E) Representative FACS plots and $(F)$ Percentages of CD3+CD4+ and CD3+ CD8+ ( $G$ and H) $T$ cells in spleen lymphocytes. Results represent mean $\pm S D\left(n=6 ;{ }^{* \star} P<0.01\right.$, $\left.{ }^{\star \star *} P<0.001\right)$. 
A

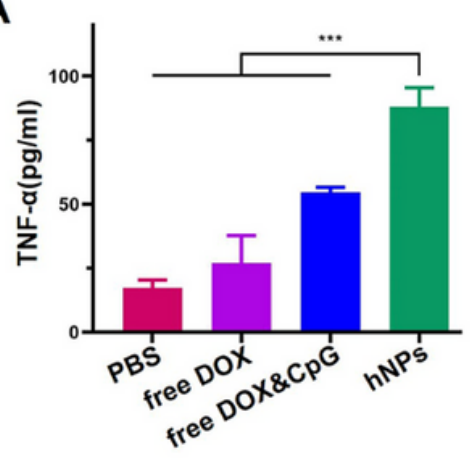

B

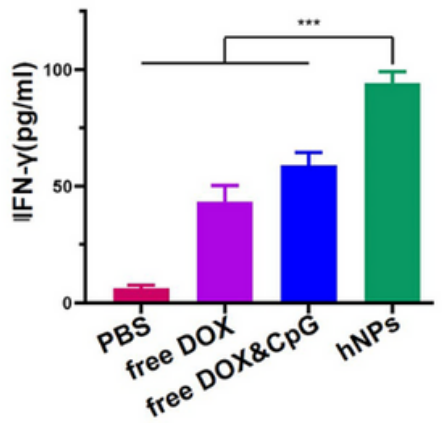

C

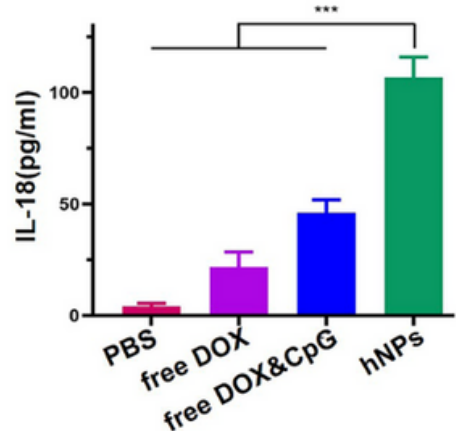

D

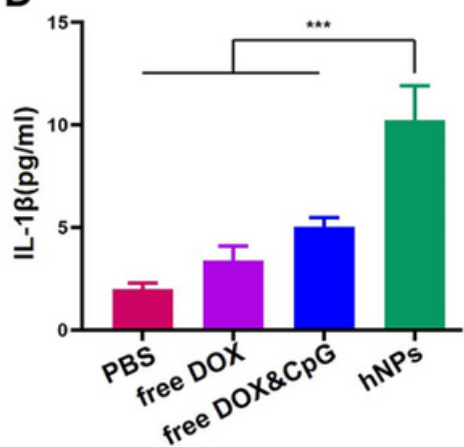

E
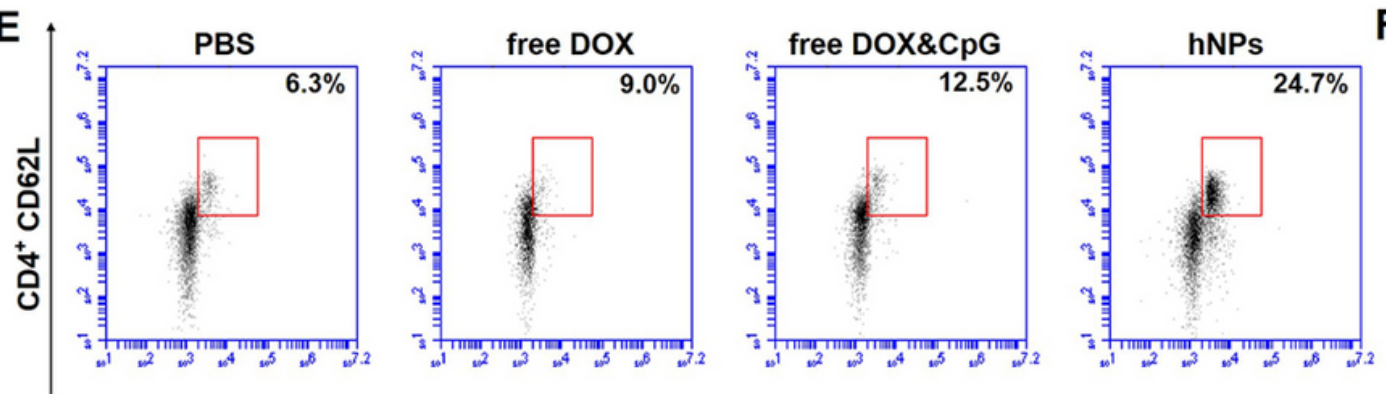

$\mathbf{F}$

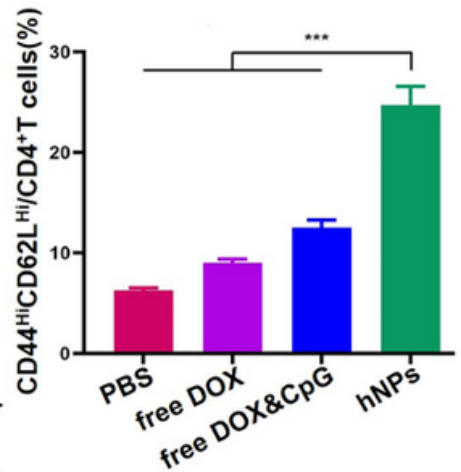

G

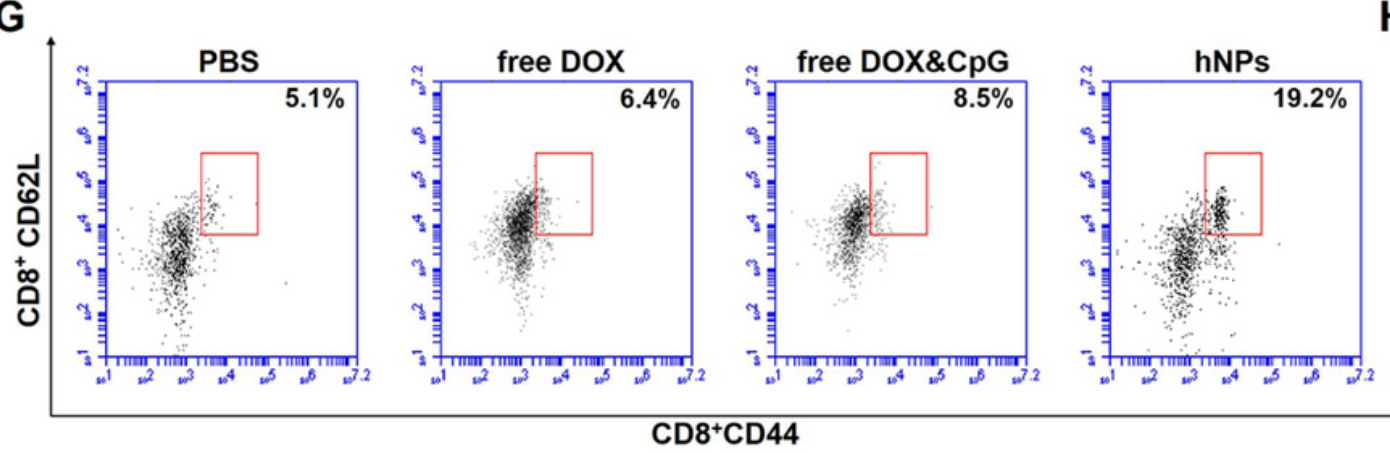

Figure 6

The cytokine expression of (A) TNF-a, (B) IFN- - , (C) IL-18 and (D) IL-1 $\beta$ in supernatant after spleen lymphocytes from various treated mice re-stimulated with E.G7-OVA fragments were analyzed using ELISA assays. (E-H) Expression of CD62L and CD44 on CD4+ or CD8+ T cells were evaluated using Flow cytometry. Results represent mean $\pm S D(n=6 ; * \star * P<0.001)$.

\section{Supplementary Files}

This is a list of supplementary files associated with this preprint. Click to download.

- Fig1GraphicalAbstract.tif

- Supplementarydata.docx 